\title{
Cloud motion vectors from MISR using sub-pixel enhancements
}

\begin{tabular}{|c|c|}
\hline Roger Davies & r.davies@auckland.ac.nz \\
\hline Akos Horvath & ahorvath@,rsmas.miami.edu, \\
\hline Catherine Moroney & Catherine.M.Moroney@jpl.nasa.gov \\
\hline Banglin Zhang $<$ & banglin@gmao.gsfc.nasa.gov \\
\hline Yanqiu Zhu & zhu@gmao.gsfc.nasa.gov \\
\hline
\end{tabular}

\begin{abstract}
The operational retrieval of height-resolved cloud motion vectors by the Multiangle Imaging Spectro Radiometer on the Terra satellite has been significantly improved by using sub-pixel approaches to coregistration and disparity assessment, and by imposing stronger quality control based on the agreement between independent forward and aft triplet retrievals. Analysis of the fore-aft differences indicates that CMVs pass the basic operational quality control $67 \%$ of the time, with rms differences -- in speed of 2.4 $\mathrm{m} / \mathrm{s}$, in direction of $17^{\circ}$, and in height assignment of $290 \mathrm{~m}$. The use of enhanced quality control thresholds reduces these rms values to $1.5 \mathrm{~m} / \mathrm{s}, 17^{\circ}$ and $165 \mathrm{~m}$, respectively, at the cost of reduced coverage to $45 \%$. Use of the enhanced thresholds also eliminates a tendency for the rms differences to increase with height. Comparison of CMVs from an earlier operational version that had slightly weaker quality control, with 6-hour forecast winds from the Global Modeling and Assimilation Office yielded very low bias values and an rms vector difference that ranged from $5 \mathrm{~m} / \mathrm{s}$ for low clouds to $10 \mathrm{~m} / \mathrm{s}$ for high clouds.
\end{abstract}

\section{Popular Summary}

Unlike most satellite instruments, Multiangle Imaging SpectroRadiometer (MISR) on the Terra satellite is a new instrument that will image Earth's climate system simultaneously at 9 different angles. One camera points toward nadir, and the others provide forward and aftward view angles, at the Earth's surface, of $26.1^{\circ}, 45.6^{\circ}, 60.0^{\circ}$, and $70.5^{\circ}$. As the instrument flies overhead, each region of the Earth's surface is successively imaged by all nine cameras. in each of four wavelengths (blue, green, red, and near-infrared).

In addition to measure the amount of sunlight that is scattered in different directions, MISR can also distinguish different types of clouds, aerosol particles, and surfaces. With the information obtained from MISR in the amount, types, and heights of clouds and the distribution of land surface cover, the heightresolved cloud motion vectors (CMVs) can be retrieved.

In this paper, we focused on improving operational retrieval algorithm of height-resolved CMVs. Subpixel approaches were used for co-registration and disparity assessment, and stronger quality control was imposed based on the agreement between independent forward and aft triplet retrievals. Analysis of the fore-aft differences indicated that CMVs passed the basic operational quality control $67 \%$ of the time, with rms differences -- in speed of $2.4 \mathrm{~m} / \mathrm{s}$, in direction of $17^{\circ}$, and in height assignment of $290 \mathrm{~m}$. The use of enhanced quality control thresholds reduced these rms values to $1.5 \mathrm{~m} / \mathrm{s}, 14^{\circ}$ and $165 \mathrm{~m}$, respectively, at the cost of reduced coverage to $45 \%$. Use of the enhanced thresholds also eliminated a tendency for the rms differences to increase with height. Comparison of CMVs from an earlier operational version that had slightly weaker quality control, with 6-hour forecast winds from the Global Modeling and Assimilation Office yielded very low bias values and an rms vector difference that ranged from $5 \mathrm{~m} / \mathrm{s}$ for low clouds to $10 \mathrm{~m} / \mathrm{s}$ for high clouds. 


\section{Cloud Motion Vectors from MISR Using Sub-pixel Enhancements}

Roger Davies

Department of Physics, The University of Auckland, New Zealand

Ákos Horváth, University of Miami, FL, USA

Catherine Moroney

Jet Propulsion Laboratory, California Institute of Technology, Pasadena, CA, USA

Banglin Zhang, Global Modeling and Assimilation Office, NASA Goddard Space Flight Center, Greenbelt, MD USA

Yanqiu Zhu, Global Modeling and Assimilation Office, NASA Goddard Space Flight Center, Greenbelt, MD USA

\section{Abstract}

\section{Introduction}

Previously [1-3], we have reported on the retrieval of cloud motion vectors (CMV) obtained by the Multiangle Imaging SpectroRadiometer (MISR) on the Terra satellite. These are derived by matching cloud reflectivity patterns from three different view angles (An, $\mathrm{B}$, and $\mathrm{D}$ cameras, corresponding to view angles of $0^{\circ}, 45^{\circ}$ and $70^{\circ}$ ), one pair at a time (i.e., An-B and B-D). The across-track and along-track disparities enter two time-dependent equations that are then solved simultaneously to separate the height and motion effects. This yields the cloud motion components parallel to and orthogonal to the satellite's direction, as well as the height of the cloud top. The retrieval is relatively noisy, and must be repeated many times over a mesoscale domain (of dimension $70.4 \mathrm{~km}$ ) to obtain a consensus motion vector from the distribution of individual retrievals performed at the $275 \mathrm{~m}$ pixel level. Experience 
with the original operational algorithm showed that it was capable of achieving a theoretical accuracy of $\sim 4 \mathrm{~m} / \mathrm{s}$, with a height resolution of $\sim 400 \mathrm{~m}$, for many mesoscale regions, but that higher errors were also common due to limited quality control. In particular, the retrievals were found to be highly sensitive to the precision to which the multiangle views were co-registered to a common reference level.

Accordingly, several improvements have since been made to the original algorithm. As described below, these improvements have led to more accurate, and far more reliable, CMVs. The off-nadir views are now rigorously co-registered to the nadir view with a sub-pixel accuracy of $\sim 0.2$ pixels, separately for each orbit, using an automated matching procedure that recognizes land-surface features or sea-ice patterns [4]. Previously, the Da camera (aft view of $70^{\circ}$ ) had a higher uncertainty in its co-registration, preventing the reliable use of the aft triplet (An-Ba-Da). With the improved co-registration of all cameras, we can now use the Da camera with confidence, and thus obtain two equivalent estimates of the mesoscale $\mathrm{CMV}$, one from the forward triplet (An-Bf-Df), and one from the aft triplet, with their agreement providing a strong measure of quality control. In addition, the distribution of $275-\mathrm{m}$ retrievals is now being interpolated at the sub-pixel level to further improve the precision of the consensus mesoscale vector.

These improvements are described in the following, followed by an assessment of the new CMV accuracy and results from a comparative study conducted by the Global Modeling and Assimilation Office (GMAO).

\section{Retrieval sensitivities to co-registration errors}

In [1], we introduced a simplified model of the wind ${ }^{1}$ retrieval problem, in

${ }^{1}$ For ease of readability, the technically correct ' $\mathrm{CMV}$ ' is often referred to simply as 
which we ignored the cross-track component of look-vectors and cloud motion, and assumed a non-rotating spherical Earth and circular obit. This elementary model takes advantage of the fact that the stereo effect is much stronger in the along-track direction than in the cross-track direction and proves helpful in investigating the sensitivity of the retrieval algorithm, at least to along-track camera misregistration. From equation 3 in [1] we can derive an estimate for the sensitivity of the retrieved along-track wind, $v_{l}$, and cloud height, $h$, to the along-track image location in the D and B cameras, $x_{D}$, and $x_{B}$, respectively. As an example, let us consider the default aftward wind retrieval camera triplet (Da-Ba-An), for which these sensitivities are as follows:

$$
\begin{gathered}
\frac{? \varpi_{\alpha}}{? \xi_{\Delta \alpha}}=\frac{\delta_{1}}{\tau \delta_{1}-\tau \delta_{2}}, \\
\frac{? \varpi_{\lambda}}{? \xi_{B \alpha}}=-\frac{\delta_{1}+\delta_{2}}{\tau \delta_{1}-\tau \delta_{2}} \\
\frac{? \eta}{? \xi_{\Delta \alpha}}=-\frac{\tau}{\tau \delta_{1}-\tau \delta_{2}} \\
\frac{? \eta}{? \xi_{B \alpha}}=\frac{\tau+\tau_{2}}{\tau \delta}-\tau \delta_{2}
\end{gathered}
$$

and

(Similar expressions can be obtained for the forward Df and Bf cameras but with opposite sign.) In the above equations, $t_{1}=\tau_{B \alpha}-\tau_{A v}, t_{2}=\tau_{\Delta \alpha}-\tau_{B \alpha}$, $d_{1}=\operatorname{Tov} \theta_{A v}-\operatorname{cov} \theta_{B \alpha}$, and $d_{2}=\operatorname{Tov} \theta_{B \alpha}-\operatorname{cov} \theta_{\Delta \alpha}$, where $t_{A n}, t_{B a}$, and $t_{D a}$ are the An, $\mathrm{Ba}$, and $\mathrm{Da}$ camera imaging times, and $\theta_{A v}, \theta_{B \alpha}$, and $\theta_{\Delta \alpha}$ are the $\mathrm{An}, \mathrm{Ba}$, and $\mathrm{Da}$

\section{'wind'.}


camera view angles. In order for the along-track wind to have a positive meridional component, its sign convention treats it as negative if it is in the same direction as the satellite motion down-track. Retrieval uncertainties resulting from an along-track coregistration uncertainty of 1 pixel $(275 \mathrm{~m})$ calculated from Eqs. 1-4 using nominal values for camera view angles and imaging times are summarized in Table 1. This shows that the retrieval uncertainty is $2-3$ times more sensitive to B camera coregistration error than it is to D camera co-registration error. This is because the $B$ image location affects both the B-D and the B-An disparities, whereas the D image location affects only the B-D disparity.

The simplified model above considers the along-track direction only. In reality, the look-vectors also have cross-track (y-axis) components that introduce a weak coupling between the along-track and cross-track wind retrievals for the general case. Consequently, a co-registration error in the along-track direction creates an error in the cross-track wind as well. Similarly, a co-registration error in the cross-track direction also has an effect on the along-track wind, although this effect is relatively small. In order to get a full picture of the various retrieval uncertainties, we performed a sensitivity analysis with the operational CMV algorithm that treats the $3 \mathrm{D}$ nature of the problem properly using ray-intersection. We applied co-registration errors of up to 1 pixel in the along-track and the cross-track directions and show the resulting retrieval errors separately for the $\mathrm{Da}$ and $\mathrm{Ba}$ cameras in Fig. 1. Retrieval uncertainties corresponding to an along-track or cross-track co-registration uncertainty of 1 pixel are also summarized in Table 2 and Table 3, respectively. Comparison of Tables 1-3 shows the following. First, the simplified model is perfectly adequate for estimating retrieval errors due to along-track misregistration. Second, retrievals are more 
sensitive to along-track misregistration than to cross-track misregistration. In fact, the effects of the latter might be neglected. Finally, the along-track wind is generally more sensitive to misregistration than is the cross-track wind.

The significance of the above findings is that in [1] we only considered retrieval errors due to finite pixel size. Such random quantization errors, however, tend to cancel out when the mean wind of a mesoscale domain is computed from a few dozen individual retrievals. Systematic errors in camera co-registration, (which tend to persist over large sections of a given orbit), on the other hand, do not cancel out and tend to bias the retrievals. Our current analysis indicates that MISR CMV retrievals are indeed sensitive to mis-registration of either the D or B cameras, with the largest retrieval errors occurring for along-track co-registration errors in the B cameras. Although co-registration errors in the B cameras are usually much smaller than in the D cameras, they can still result in significant CMV and height errors due to their enhanced effect on the retrievals. Therefore, we have implemented an algorithm that, in the presence of visible land-surface or sea-ice features, improves not only the D but also the B camera co-registration. This is performed at the sub-pixel level, separately for each orbit [4].

In addition to these co-registration enhancements, the analysis of the measured disparities has also been improved. Previously, along-track and cross-track disparities retrieved over a given domain were sorted into a $2 \mathrm{D}$ histogram, and then the final mesoscale wind was computed from the most populated histogram bin. Because the MISR stereo matchers have no sub-pixel capability, this meant that the modal alongtrack and cross-track disparities were always quantized as integer values. In the updated algorithm, the final mesoscale wind is calculated as the weighted average of 
the most populated bin and the surrounding bins. This yields more precise (floating point) values for the modal disparities, even though the input disparities are still integers. Experience with the new algorithm indicates that the enhanced coregistration and modal disparity computation contribute about equally to reducing the rms errors in the updated CMVs, with the co-registration error being mainly responsible for the bias error.

\section{Comparison of forward and aft CMV retrievals Quality Control}

In addition to the above enhancements, a more rigorous quality control has now been implemented. The main quality check is obtained by comparing the forward triplet retrieval with that from the aft triplet. If any one of the fore-aft retrievals of height, CMV direction, or either wind component, differs by a basic threshold, then the quality is labeled 'bad'. Since directionality loses its significance as the wind speed drops to zero, the directionality threshold is applied only to wind speeds $>2 \mathrm{~m} / \mathrm{s}$. For operational purposes, the basic thresholds are chosen empirically with fairly broad tolerance, with the intention of removing gross blunders due to either co-registration error or stereo mismatches. No attempt is made to further salvage 'bad' CMVs, although this may be possible for special cases that can be studied at greater depth. This yields the basic data set of operationally retrieved 'good' CMVs that are used in the subsequent comparison with the GMAO.

Intended for research, rather than for basic operational purposes, a subset of improved quality, or 'better', CMVs is also obtained from the 'good' CMV data set by setting enhanced thresholds for the height and wind components. For a 'good' CMV to be labeled 'better' it must satisfy at least two of the enhanced thresholds. The basic and 
enhanced thresholds are summarized in Table 4.

\section{Analysis of Differences}

To assess the internal consistency of the CMV retrievals, the forward and aft differences were analyzed over a randomly chosen set of 10 orbits. This produced sufficient data for representative statistics. The frequency distributions of the differences between the forward and aft retrievals are shown in Figs. 2-4 for, respectively, the scalar wind speed, the wind direction, and the wind height. The 'better' distributions are narrower than the 'good' distributions, as expected from their definition, and both distributions are relatively unbiased and symmetric. The shape of the distributions helps to indicate the role of the thresholds, as these cut off the tails of the overall distributions. For most of these, the effect is simply to remove outliers from the tail of the distribution. For the 'better' wind-height differences, the enhanced height difference threshold probably limits the distribution a little too strongly for the rejected data to be called outliers.

The mean and rms of the fore-aft wind-speed, wind-direction, and wind-height differences, together with the vector rms difference, and the overall coverage, are summarized in Table 5. In the absence of a fore-aft quality control, the rms differences are quite high due to the presence of blunders. The low wind-speed bias in the absence of quality control shows simply that the blunders are not preferentially positive or negative. Some of these blunders could be removed by other techniques, as by examining the original disparity histograms, which would eliminate about $10 \%$ of the data and reduce the rms speed error to about $13 \mathrm{~m} / \mathrm{s}$. However, the fore-aft quality control is far more effective. This quality control comes at the expense of eliminating 
about one third of the total coverage, but some of this includes clear regions, multileveled clouds, and featureless clouds for which the triplet stereo approach is not effective anyway.

The difference between 'good' and 'better', in terms of overall statistics, is not profound. By using the enhanced thresholds, the rms speed difference reduces to 1.5 $\mathrm{m} / \mathrm{s}$ with a coverage that is still almost half of the data. It would of course be possible to reduce this difference further, eliminating more data, but for the following we simply address the 'good', or operational product.

\section{Comparison with the Global Modeling and Assimilation Office}

The MISR CMVs were also compared against the 6-hour model forecast winds from version 4.03 of the GEOS data assimilation and forecast systems of the GMAO. A 6week data set starting 1 September 2003 was used for this comparison. The operational MISR product for 'good' winds, version 4.0, included the sub-pixel enhancements mentioned above, but used a slightly earlier version of the quality control, with tighter basic thresholds for the along-track wind difference, and no across-track, height, or direction thresholds. These thresholds are somewhat correlated, so the overall differences in quality are not very great. The summary statistics for the fore-aft differences for the same version of processing used in the comparison set are also given in Table 5. These values are slightly higher than for the current 'good' winds.

The GMAO analyses are a combination of information from the forecast model and several types of observations. The latter include wind and mass profiles from radiosondes, conventional surface observations, aircraft measurements, surface wind 
data from satellite scatterometers, geostationary satellite AMVs (atmospheric motion vectors that may include both cloud and water vapor features), mass and humidity data from infrared and microwave satellite sounders, and total precipitable water from passive microwave imagers.

The results of this comparison are summarized in Table 5. The bias difference appears to be very low, and is nearly zero for the low-level and mid-level winds. The rms vector difference is $\sim 5 \mathrm{~m} / \mathrm{s}$ for low-level winds, rising with height. The rms uncertainty in the GMAO forecast winds, which are short-term forecasts issued from GMAO analyses, is not known definitively, but appears to rise with height as the mean wind speed increases. The increased rms difference between MISR and GMAO with height was not expected to be as large an effect, however, since the MISR technique is in principle independent of height. However, on stratifying the fore-aft differences with height for version 4.0 of the operational 'good' winds, these also increased with height, as shown in Fig. 5. Part of the explanation is that the crosstrack wind component increases with height, and no quality control was being implemented on this in version 4.0. In the current version (4.1), most of the height dependence of the vector rms differences disappears, and for the 'better' winds it disappears completely, also shown in Fig. 5. At low levels, which dominate the overall data set due to their greater population, the fore-aft differences in both versions of the 'good' winds indicate a vector rms difference of about $3.5 \mathrm{~m} / \mathrm{s}$. Since the difference with the GMAO forecast winds is about $5.1 \mathrm{~m} / \mathrm{s}$, there remains about $3.7 \mathrm{~m} / \mathrm{s}$ of difference (subtracting in quadrature) that is not explained. Some of this is attributable to the uncertainty in the forecast winds. For upper-level winds, the unexplained difference rises to about $7.2 \mathrm{~m} / \mathrm{s}$, albeit for much fewer samples.

9 of 19 , printed $11 / 30 / 06$, draft version prior to submission 


\section{Discussion}

With the implementation of the changes described here, notably the use of sub-pixel co-registration and a fore-aft quality control, the operational MISR wind product has likely reached full maturity. With $67 \%$ coverage, the rms speed error is $\sim 2.4 \mathrm{~m} / \mathrm{s}$, the rms direction error for winds over $2 \mathrm{~m} / \mathrm{s}$ is $\sim 17^{\circ}$, and the rms height error of the CMVs is $\sim 300 \mathrm{~m}$. These errors appear to lack measurable bias. Comparison with the GMAO forecast winds over an extensive data set also shows very low bias. The rms vector speed differences with the GMAO rise from $\sim 5$ to $\sim 10 \mathrm{~m} / \mathrm{s}$ from low to high troposphere. Part of the difference in the upper troposphere is attributable to a weaker quality control in the MISR operational version compared, that has since been improved. Part of the difference remains unexplained, and may be due in part to the higher wind speeds and greater wind shear that exists in the upper troposphere. For these, the height assignment of the CMVs plays a crucial role. By providing a geometrically based height assignment (with known uncertainty), the MISR CMVs. are insensitive to assumptions about the atmospheric temperature profile, but the comparison with other techniques, done in pressure coordinates, will introduce some uncertainty.

From the perspective of producing a superior quality product, the 'better' winds quality control yields a wind speed $\mathrm{rms}$ of $\sim 1.5 \mathrm{~m} / \mathrm{s}$ with a height uncertainty of $\sim 165$ $m$ that may be useful for in-depth dynamical case studies.

Future plans include investigating the impact of these winds, especially from data sparse areas, on forecast skill.

10 of 19 , printed 11/30/06, draft version prior to submission 
Acknowledgements

Part of this research was conducted when R. Davies and Á. Horváth were at the Jet Propulsion Laboratory, under a contract from the National Aeronautics and Space Administration. The MISR data were supplied by the NASA Langley Research Center Atmospheric Science Data Center. D.J. Diner, V. Jovanovic, and L-P. Riishojgaard are thanked for useful discussions.

\section{References}

[1] Á. Horváth and R. Davies, Feasibility and error analysis of cloud motion wind extraction from near-simultaneous multiangle MISR measurements, Journal of Atmospheric and Oceanic Technology, 18, 591-608, 2001.

[2] Á. Horváth and R. Davies, Simultaneous retrieval of cloud motion and height from polar-orbiter multiangle measurements, Geophysical Research Letters, 28, 2915$2918,2001$.

[3] C. Moroney, Á. Horváth and R. Davies, Use of stereo-matching to coregister multiangle data from MISR, IEEE Trans. Geosci. Remote Sensing, 40, 1541-1546, 2004.

[4] V. Jovanovic, C. Moroney and R. Davies, this issue, 2006. 
Table 1. Retrieval uncertainty in along-track wind, $v_{l}$, and cloud height, $h$, due to a $1-$ pixel uncertainty $(\Delta \xi=275 \mathrm{~m})$ in $\mathrm{Da}$ or Ba along-track co-registration as determined from the simplified model.

\begin{tabular}{ccc}
\hline & $\mathrm{Da}$ & $\mathrm{Ba}$ \\
\hline$\frac{? \varpi_{n}}{? \xi} \Delta \xi$ & $-5.7 \mathrm{~m} / \mathrm{s} /$ pixel & $15.6 \mathrm{~m} / \mathrm{s} /$ pixel \\
$\frac{? \eta}{? \xi} \Delta \xi$ & $507 \mathrm{~m} /$ pixel & $-1132 \mathrm{~m} /$ pixel \\
\hline
\end{tabular}

Table 2. Retrieval uncertainty in along-track wind, $v_{l}$, cross-track wind, $v_{c}$, and cloud height, $h$, due to a 1-pixel uncertainty $(\Delta \xi=275 \mathrm{~m})$ in Da or Ba along-track coregistration as determined from the operational ray-intersection algorithm.

\begin{tabular}{ccc}
\hline & $\mathrm{Da}$ & $\mathrm{Ba}$ \\
\hline$\frac{? w_{n}}{? \xi} \Delta \xi$ & $-5.6 \mathrm{~m} / \mathrm{s} /$ pixel & $15.5 \mathrm{~m} / \mathrm{s} /$ pixel \\
$\frac{? \varpi_{x}}{? \xi} \Delta \xi$ & $-1.4 \mathrm{~m} / \mathrm{s} /$ pixel & $3.4 \mathrm{~m} / \mathrm{s} /$ pixel \\
$\frac{? \eta}{? \xi} \Delta \xi$ & $519 \mathrm{~m} /$ pixel & $-1159 \mathrm{~m} /$ pixel \\
\hline
\end{tabular}

Table 3. Retrieval uncertainty in along-track wind, $v_{l}$, cross-track wind, $v_{c}$, and cloud height, $h$, due to a 1-pixel uncertainty ( $\Delta \psi=275 \mathrm{~m}$ ) in Da or Ba cross-track coregistration as determined from the operational ray-intersection algorithm.

\begin{tabular}{|l|c|c|}
\hline & $\mathrm{Da}$ & $\mathrm{Ba}$ \\
\hline$\frac{? \sigma_{\chi}}{? \psi} \Delta \psi$ & $-1.1 \mathrm{~m} / \mathrm{s} /$ pixel & $2.0 \mathrm{~m} / \mathrm{s} /$ pixel \\
\hline$\frac{? \varpi_{x}}{? \psi} \Delta \psi$ & $0.9 \mathrm{~m} / \mathrm{s} /$ pixel & $1.0 \mathrm{~m} / \mathrm{s} /$ pixel \\
\hline$\frac{? \eta}{? \psi} \Delta \psi$ & $72 \mathrm{~m} /$ pixel & $-159 \mathrm{~m} /$ pixel \\
\hline
\end{tabular}


Table 4. Quality control thresholds applied to the component differences between mesoscale CMV retrievals obtained separately using forward triplet views (An-Bf-Df) and aft triplets (An-Ba-Da).

\begin{tabular}{|c|c|c|}
\hline & $\begin{array}{c}\text { Basic Thresholds } \\
\text { (all must be satisfied for } \\
\text { 'good' winds) }\end{array}$ & $\begin{array}{c}\text { Enhanced Thresholds } \\
\text { (two or more must be } \\
\text { satisfied for 'better' } \\
\text { winds) }\end{array}$ \\
\hline NS wind component & $10 \mathrm{~m} / \mathrm{s}$ & $3 \mathrm{~m} / \mathrm{s}$ \\
\hline EW wind component & $3 \mathrm{~m} / \mathrm{s}$ & $1 \mathrm{~m} / \mathrm{s}$ \\
\hline Height & $1000 \mathrm{~m}$ & $300 \mathrm{~m}$ \\
\hline Direction & $45^{\circ}$ & not used \\
\hline
\end{tabular}

Table 5. Summary statistics for the differences between fore and aft CMV retrievals using the current basic and enhanced thresholds, as well as for the earlier basic thresholds used in the GMAO comparison.

\begin{tabular}{|l|c|c|c|c|}
\hline & All & 'good' & 'better' & 'good' (GMAO) \\
\hline Number & 10,237 & 6,866 & 4,602 & 6,295 \\
\hline Coverage & $100 \%$ & $67 \%$ & $45 \%$ & $61 \%$ \\
\hline Speed bias (m/s) & -0.10 & -0.20 & -0.14 & -0.17 \\
\hline Speed rms (m/s) & 19.1 & 2.4 & 1.5 & 2.7 \\
\hline Direction bias & $3.1^{\circ}$ & $0.9^{\circ}$ & $0.5^{\circ}$ & $2.2^{\circ}$ \\
\hline Direction rms & $55^{\circ}$ & $17^{\circ}$ & $14^{\circ}$ & $24^{\circ}$ \\
\hline Vector rms (m/s) & 23.2 & 3.7 & 2.2 & 3.9 \\
\hline Height bias (m) & 133 & 38 & 18 & 30 \\
\hline Height rms (m) & 2,102 & 291 & 165 & 649 \\
\hline
\end{tabular}

Table 6. Comparison of MISR wind retrievals with forecast winds from the Global Modeling and Assimilation Office. 


\begin{tabular}{|c|c|c|c|}
\hline & $\begin{array}{c}\text { low-level } \\
(>700 \mathrm{hPa})\end{array}$ & $\begin{array}{c}\text { mid-level } \\
(400-700 \mathrm{hPa})\end{array}$ & $\begin{array}{c}\text { high-level } \\
(<400 \mathrm{hPa})\end{array}$ \\
\hline speed bias $(\mathrm{m} / \mathrm{s})$ & 0.09 & -0.02 & 1.01 \\
\hline $\begin{array}{c}\text { rms vector difference } \\
(\mathrm{m} / \mathrm{s})\end{array}$ & 5.1 & 7.4 & 10.5 \\
\hline $\begin{array}{c}\text { mean speed }(\mathrm{m} / \mathrm{s}) \\
\text { normalized rms }\end{array}$ & 8.6 & 12.1 & 24.3 \\
\hline $\begin{array}{c}\text { vector difference } \\
(\mathrm{m} / \mathrm{s})\end{array}$ & 0.59 & 0.62 & 0.43 \\
\hline $\begin{array}{c}\text { number of } \\
\text { observations }\end{array}$ & 70,091 & 12,442 & 2,631 \\
\hline
\end{tabular}



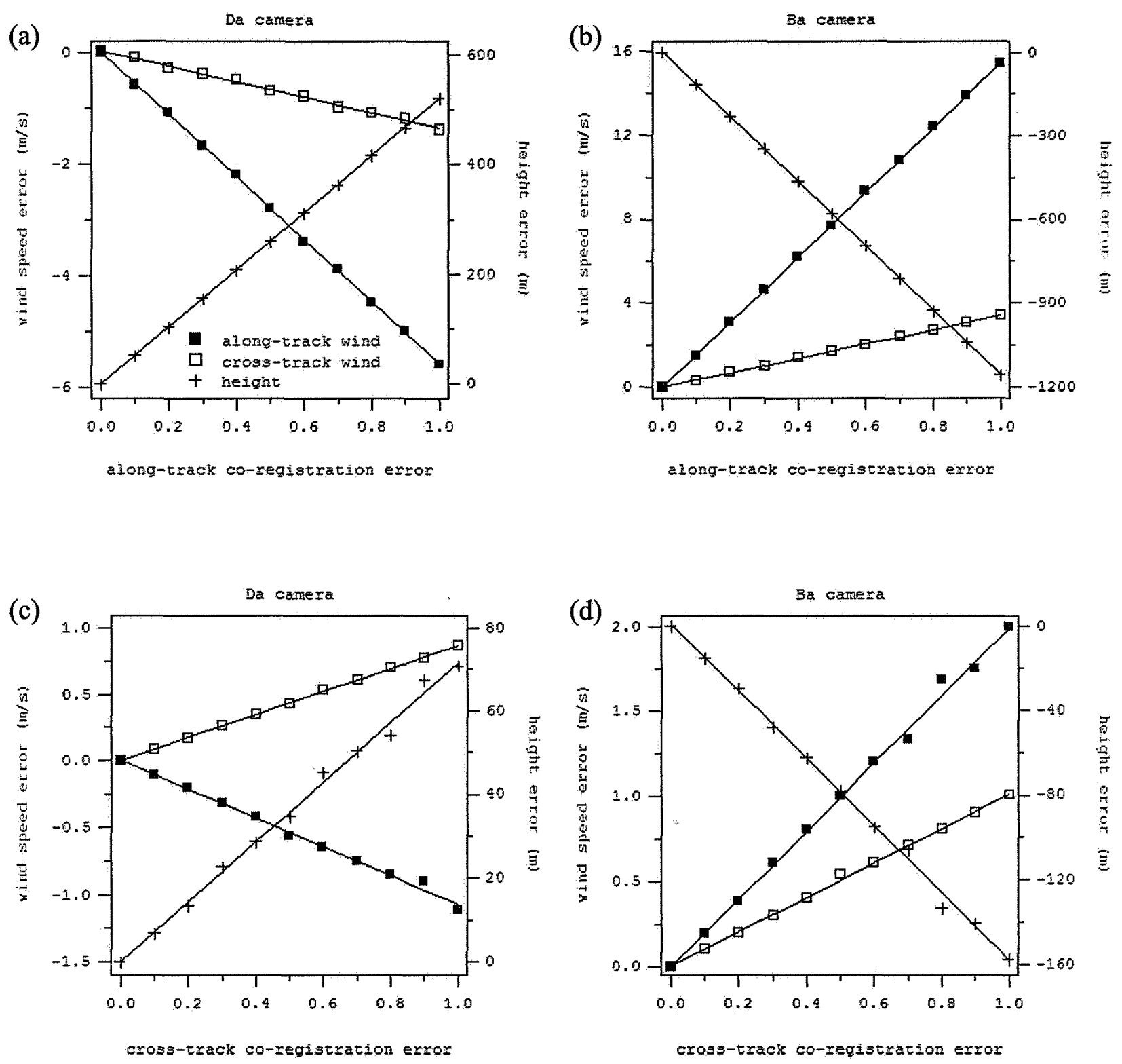

Figure 1. Retrieval error in along-track wind (solid boxes), cross-track wind (empty boxes), and cloud height (plus signs) versus co-registration error as determined from the operational ray-intersection algorithm: (a) Da along-track misregistration, (b) $\mathrm{Ba}$ along-track misregistration, (c) Da cross-track misregistration, and (d) Ba cross-track misregistration. Note that solid lines are linear fits, misregistration is given in $275-\mathrm{m}$ pixels, and the along-track and cross-track winds are positive towards north and east, respectively. 


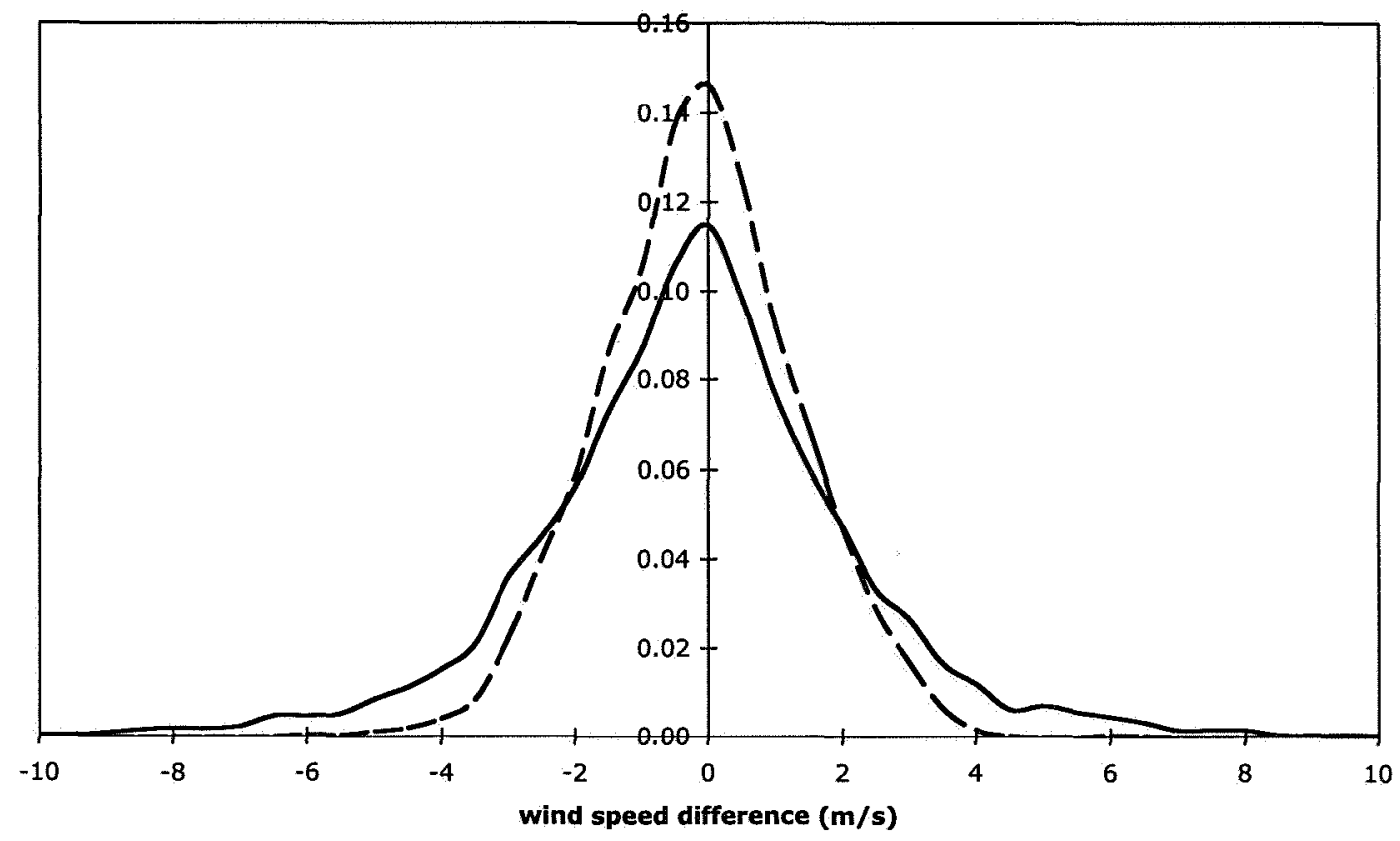

Figure 2. Frequency distribution of CMV speed differences between the forward and aft triplet retrievals, for cases that satisfy the quality control at a level of 'good' (solid) or 'better' (dashed). 


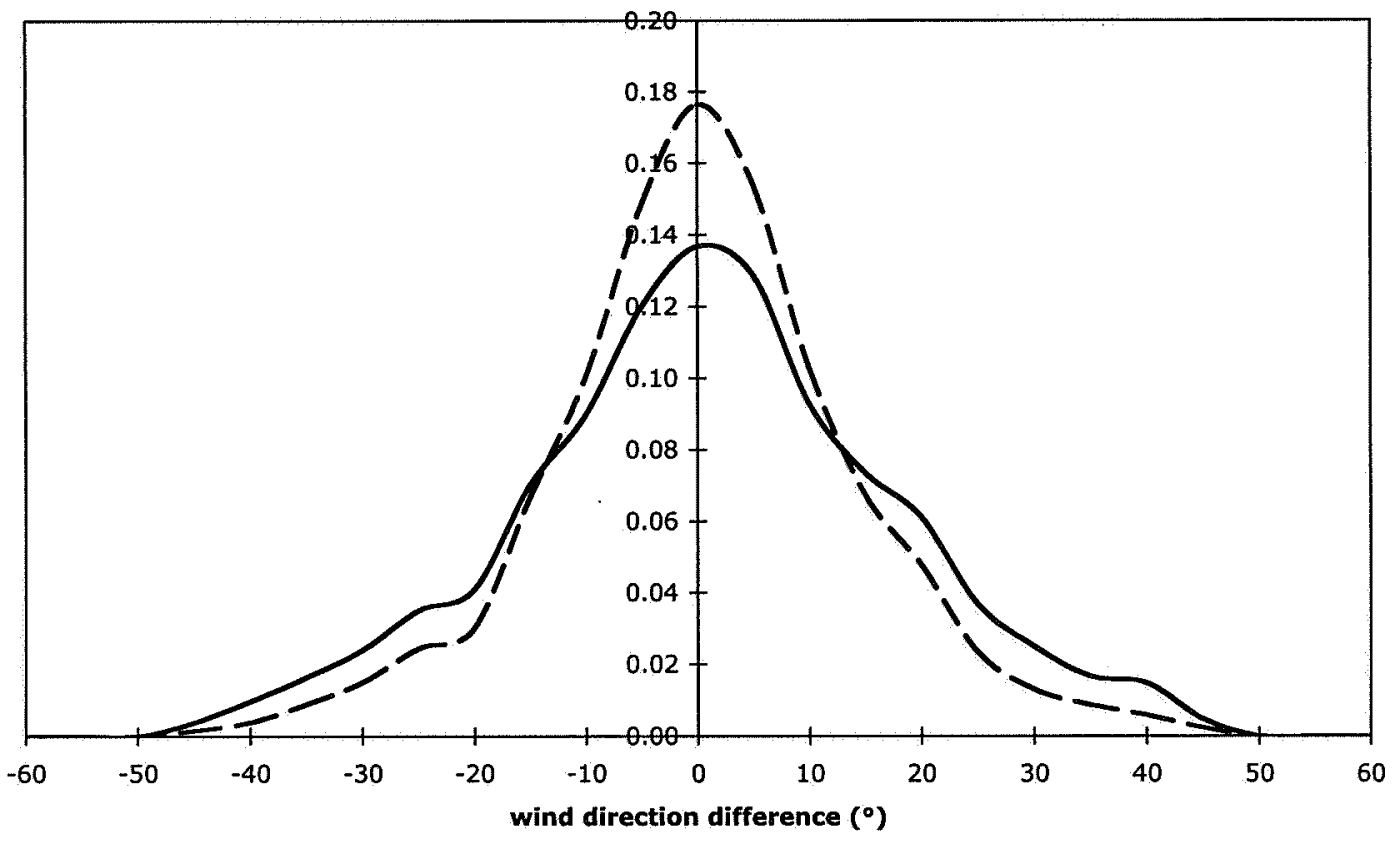

Figure 3. Frequency distribution of CMV direction differences between the forward and aft triplet retrievals, for cases that satisfy the quality control at a level of 'good' (solid) or 'better' (dashed). 


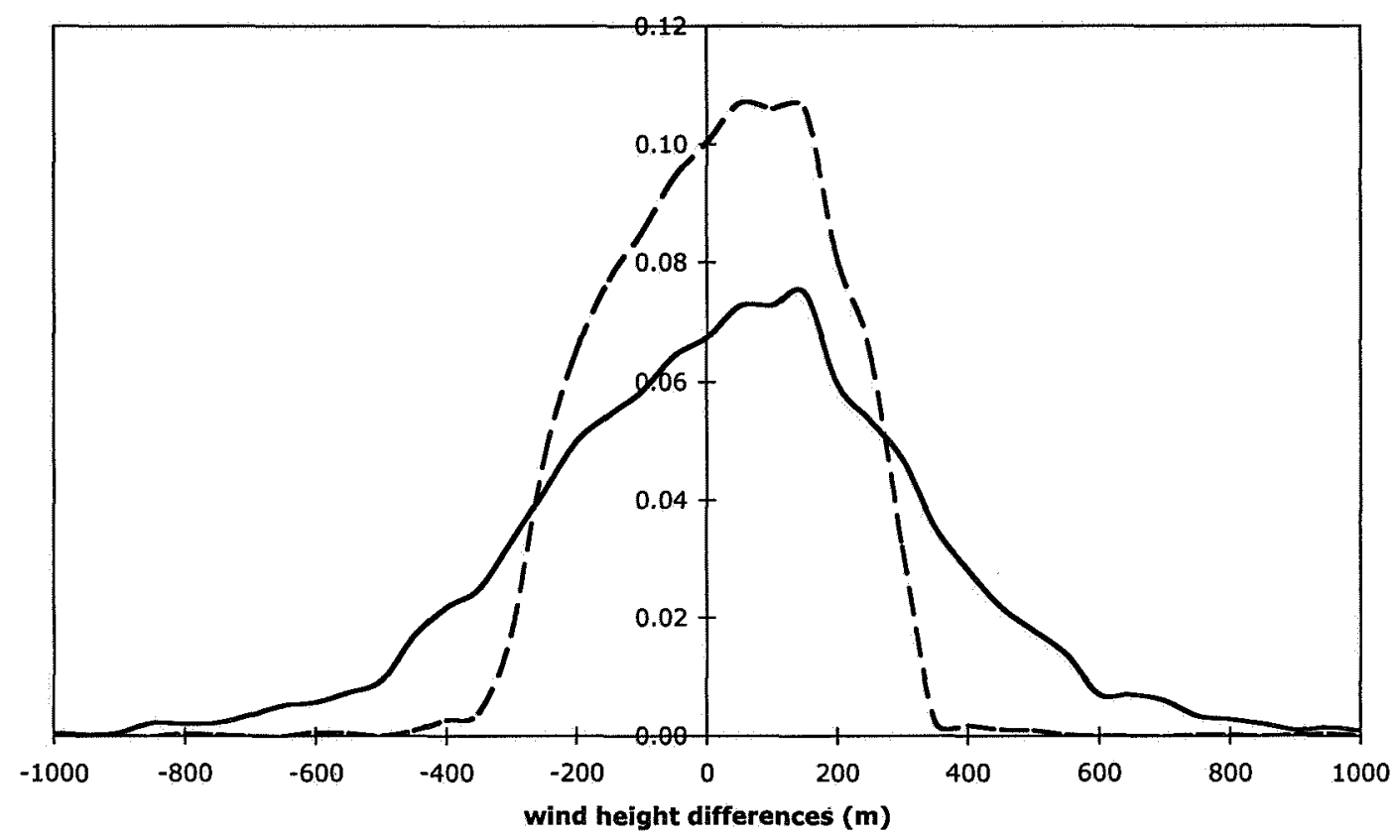

Figure 4. Frequency distribution of CMV height differences between the forward and aft triplet retrievals, for cases that satisfy the quality control at a level of 'good' (solid) or 'better' (dashed). 


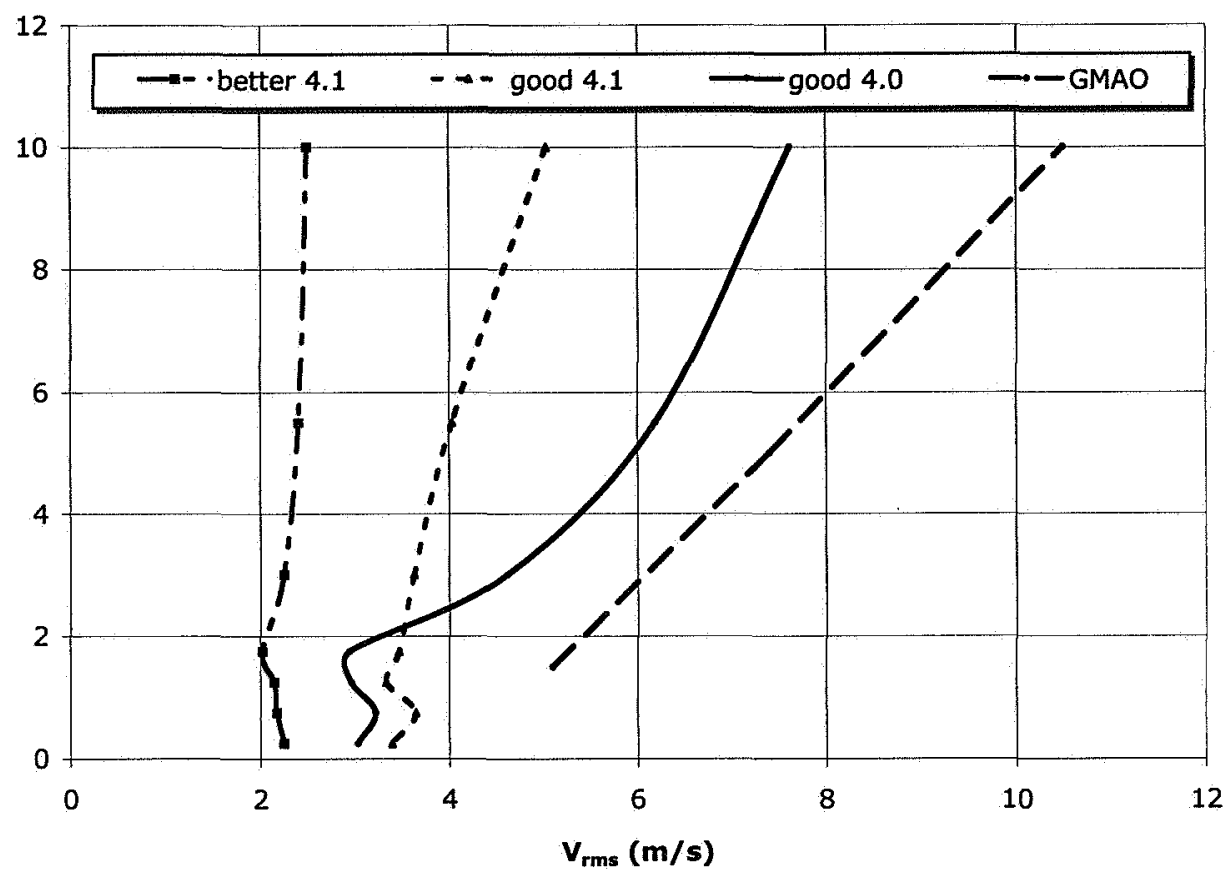

Figure 5. Vector rms speed differences as a function of height. From left to right: 'better' fore-aft differences for version 4.1; 'good' fore-aft differences for version 4.1; 'good' fore-aft differences for version 4.0; differences between MISR 'good' version 4.0 and GMAO forecast winds. 